Bundesgesundheitsbl $2011 \cdot 54: 867-874$ DOI 10.1007/s00103-011-1298-x

Online publiziert: 28. Juni 2011

(c) Springer-Verlag 2011

\author{
H. Uphoff' - S. Geis ${ }^{1,2}$ - A. Wirtz ${ }^{3}$ A.M. Hauri ${ }^{1}$ \\ ${ }^{1}$ Hessisches Landesprüfungs- und Untersuchungsamt \\ im Gesundheitswesen, Dillenburg \\ ${ }^{2}$ Robert Koch-Institut, Berlin \\ ${ }^{3}$ Hessisches Sozialministerium, Wiesbaden
}

\title{
Zeitnahe Erfassung und Übermittlung von Todesfällen in Hessen
}

\author{
Eine erste Einschätzung zur \\ Influenza-A/H1N1v-Pandemie
}

Die Erfassung von Todesfällen ist ein wichtiges epidemiologisches Instrument, um den Verlauf, das Ausmaß, betroffene Altersgruppen und die Schwere von Gesundheitsgefahren, die zu einer erkennbaren Veränderung der Mortalität führen, einschätzen zu können. International gibt es zahlreiche Beispiele für eine sinnvolle Nutzung zeitnaher Analysen geeigneter Sterbedaten. Besonders deutlich wurde der Bedarf nach einer kontinuierlichen zeitnahen Analyse von Sterbedaten nach der Hitzewelle 2003 und im Rahmen der Influenza-Surveillance und Pandemieplanung $[1,2,3,4,5,6]$. Auch die Analyse der Sterbefalldaten nach einer Chickungunia-Epidemie auf Reunion 2005/06 erbrachte Hinweise auf eine damit in $\mathrm{Zu}$ sammenhang stehende Übersterblichkeit und somit wichtige Erkenntnisse zur Bewertung der Erkrankungslast $[1,7]$.

In Deutschland sind weder auf Landes- noch auf Bundesebene geeignete Daten oder Auswertungen zur zeitnahen Erfassung von Todesfällen zugänglich. So sind auch während intensiver Influenza-Epidemien keine Schätzungen zur Übersterblichkeit verfügbar $[8,9]$. Ferner bieten die derzeit vorliegenden Statistiken eine unzureichende zeitliche Auflösung nur bis zu Monatsintervallen [10, 11, 12]. Zu Schätzungen der Exzesstodesfälle während der Hitzewelle 2003 wurden da- her lokale Datensätze eines Standesamtes oder Sondererhebungen genutzt, um kürzere Zeitintervalle betrachten zu können $[13,14,15]$.

Aus diesen Gründen wurde in Hessen das im Folgenden vorgestellte Surveillance-System zur Zeitnahen Erfassung von Todesfällen in Hessen (ZETH) aufgebaut.

\section{Gesetzliche Grundlagen zur Todesfallerfassung und Meldewege}

Die Registrierung von Todesfällen ist in Deutschland im Personenstandsgesetz (PStG) und darüber hinaus in Gesetzen und Verordnungen der Länder geregelt. Die Datensätze und Meldewege sind damit bundesweit einheitlich. Mit der 2009 in Kraft getretenen Reform des PStG wurde bundesweit eine elektronische Datenerfassung und Datenarchivierung festgelegt (mit Übergangsfristen bis zum Jahr 2013, $\$ 75$ ). In Hessen wurde die elektronische Datenerfassung und Übermittlung im Rahmen des Projektes e-Government gefördert. Daher ist in den Kommunen bereits jetzt der Anteil der elektronisch durchgeführten Datenverwaltung hoch.

Entsprechend $\$ 32$ PStG müssen Todesfälle dem Standesamt, das für den Be- reich, in dem die Person verstorben ist, spätestens am auf den Todestag folgenden Werktag gemeldet werden. Im Standesamt werden alle Todesfälle registriert, geprüft $(\$ 36 \mathrm{PStG})$ und baldmöglichst beurkun$\operatorname{det}(\$ 37$ PStG). Die beurkundeten Fälle werden spätestens zum 15. des Folgemonats an das Statistische Landesamt (HSL), die zuständigen Einwohnermeldeämter und die Gesundheitsämter übermittelt. Die Übermittlung erfolgt teils elektronisch, teils auf Papierbasis. Die in den Standesämtern erhobenen Daten werden a) für die Bevölkerungsfortschreibung und b) für die Todesursachenstatistik verwendet. Die hierfür benötigten Daten werden auf unterschiedlichen Wegen an die Statistischen Landesämter übermittelt und dort von unterschiedlichen Abteilungen bearbeitet.

Bevölkerungsfortschreibung. Für die Bevölkerungsfortschreibung werden in Hessen die Todesfälle in über 90\% der Standesämter elektronisch erfasst und an das Sachgebiet „Bevölkerung“ des HSL weitergeleitet. Zusätzlich erfolgt eine, ebenfalls überwiegend elektronische, Übermittlung an die Einwohnermeldeämter.

Todesursachenstatistik. Für die Todesursachenstatistik wird der vertrauli- 
Tab. 1 Bezeichnung, Format und Beispiele der in der Hessischen Todesfalldatei erfassten Items

\begin{tabular}{|c|c|c|}
\hline Bezeichnung & Format & Beispiel \\
\hline Endedatum & Ttmmijjjj & 13.10 .2006 \\
\hline $\begin{array}{l}\text { Versterbe-Land } \\
\text { (Kennziffer) }\end{array}$ & Zahl & 06 \\
\hline $\begin{array}{l}\text { Versterbe-Land } \\
\text { (Klartext) }\end{array}$ & String & Hessen \\
\hline $\begin{array}{l}\text { Versterbe-Kreis } \\
\text { (Kennziffer) }\end{array}$ & Zahl & 411 \\
\hline $\begin{array}{l}\text { Versterbe-Kreis } \\
\text { (Klartext) }\end{array}$ & String & Darmstadt \\
\hline $\begin{array}{l}\text { Geschlecht (Kenn- } \\
\text { ziffer) } 1 \text { oder } 2\end{array}$ & Zahl & 1 \\
\hline Geschlecht (Klartext) & String & Männlich \\
\hline Sterbedatum & tt.mm.jjjj & 15.03.2006 \\
\hline Geburtsdatum & mm.jjjj & 08.1934 \\
\hline $\begin{array}{l}\text { Bundesland des } \\
\text { Wohnortes } \\
\text { (Kennziffer) }\end{array}$ & Zahl & 06 \\
\hline $\begin{array}{l}\text { Bundesland des } \\
\text { Wohnortes (Klartext) }\end{array}$ & String & Hessen \\
\hline $\begin{array}{l}\text { Kreis des Wohnortes } \\
\text { (Kennziffer) }\end{array}$ & Zahl & 412 \\
\hline $\begin{array}{l}\text { Kreis des Wohnortes } \\
\text { (Klartext) }\end{array}$ & String & Frankfurt \\
\hline
\end{tabular}

che Teil der Leichenschauscheine an die Gesundheitsämter noch ausschließlich auf Papierbasis übermittelt. Nach seiner Durchsicht leiten die Gesundheitsämter diesen an die Erfassungsstelle der Todesursachenstatistik im HSL und gegebenenfalls an das Krebsregister weiter. Im HSL erfolgt die digitale Erfassung und ICDCodierung der Todesfälle für die Todesursachenstatistik.

Sowohl die Todesursachenstatistik als auch die Bevölkerungsfortschreibung der Länder werden im Statistischen Bundesamt zu Bundes-Statistiken zusammengefasst. Diese Statistiken sind eher als Jahresstatistiken angelegt, und geringere zeitliche Auflösungen als nach Monaten werden routinemäßig nicht zur Verfügung gestellt [12].

Im Folgenden werden der Aufbau und die Struktur eines hessenweiten Erfassungssystems beschrieben, das eine wochenaktuelle Auswertung aller beurkundeten und elektronisch übermittelten Todesfälle durch das Hessische Landesprüfungs- und Untersuchungsamt im Gesundheitswesen (HLPUG) ermöglicht. Dargestellt werden die zur Verfügung stehenden Daten, Meldewege und -zeiten so- wie erste Erfahrungen mit deren Zusammenfassung und Bewertung während der Influenza- $\mathrm{A} / \mathrm{H}_{1} \mathrm{~N}_{1 v}-$ Pandemie.

\section{Aufbau und Struktur des ZETH}

\section{Auswahl der Datengrundlage: Bevölkerungsstatistik oder Todesursachenstatistik?}

In Gesprächen zwischen Vertretern des HSL, des Hessischen Sozialministeriums (HSM), des Hessischen Innenministeriums (HMdI) und des HLPUG wurde deutlich, dass einzig die Beschleunigung eines bereits bestehenden Meldeweges als Basis für das ZETH infrage kam. Die Todesursachenstatistik wurde hierfür aus folgenden Gründen als wenig geeignet erachtet:

- Standesämter geben die Daten ausschließlich in Papierform an die Gesundheitsämter weiter, und - die Gesundheitsämter geben die Daten ausschließlich in Papierform an das HSL weiter.

Damit vergeht bis zur zentralen digitalen Erfassung und ICD-Kodierung der Daten im HSL eine zu lange Zeit.

Die Abfrage von Daten der Einwohnermeldeämter, die meist von kommunalen Rechenzentren in privater Dienstleistung verwaltet werden, wurde aus Kostengründen verworfen.

Aus diesen Gründen wurde beschlossen, die Weiterleitung der Standesamtsdaten an das HSL im Rahmen der Bevölkerungsstatistik von einer monatlichen auf eine wöchentliche zu beschleunigen.

\section{Rechtliche Grundlage der wöchentlichen Übermittlung}

Mit einem Erlass des HMdI wurden alle hessischen Standesämter ersucht, ab der 24. Kalenderwoche 2008 Sterbedaten elektronisch bis jeweils Dienstag 10:0o Uhr der Woche nach dem Meldeeingang an das HSL zu übermitteln. Die elektronische Übermittlung erfolgt in Hessen seit 2006 über das „Portal Internet-Datenerhebung im Verbund (IDEV) “ (www.statistik-portal.de/statistik-portal/ datenOnline.asp).

\section{Beschreibung der Dateien und Variablen}

Das HSL stellt dem HLPUG eine datenschutzkonforme Zusammenstellung der Todesfälle über zwei Dateien zur Verfügung:

Todesfalldatei. Eine Datei - die Todesfalldatei - enthält die als Einzelfall erfassten Sterbedaten mit den in $\bullet$ Tab. 1 aufgeführten Feldern.

Kontrolldatei. Des Weiteren wird eine „Kontrolldatei“ mit einer Auflistung aller Standesämter übermittelt, die Daten in die Todesfalldatei eingebracht haben. Die Kontrolldatei enthält auch die Summe der jeweils übermittelten Fälle und das jeweilige Erstellungsdatum der Exportdatei (Variablenname: Endedatum) sowie die Standesämter, die eine „Nullmeldung“ übermittelt haben.

Das HSL führt außer einer Kontrolle doppelter Datensätze keine systematische Prüfung der an das HLPUG zu übermittelnden Daten durch. Die im Folgenden dargestellten Auswertungen schließen Daten bis zum 20.4.2010 ein.

\section{Vergleichsdaten zu den Vorjahren}

Dem HLPUG stehen die elektronisch übermittelten Todesfallzahlen (• Tab. 1) ab dem 1.1.2006 als vorläufige Zahlen zur Verfügung. Weiterhin wurde eine monatsweise aggregierte Datei der Todesfälle 2005 stratifiziert nach Standesämtern sowie eine Liste aller Standesämter mit Telefonnummern und Ansprechpartnern zur Verfügung gestellt.

\section{Anpassung der}

Software-Programme in den Standesämtern und dem HSL

In den Exportdatensatz wurde zusätzlich das Feld „Endedatum“ eingefügt (nach Abstimmung mit den Referenten der Fachabteilungen „Bevölkerungsfortschreibung" der Statistischen Landesämter). Dieses Feld dokumentiert das Datum, zu dem die Datenauslese im jeweiligen Standesamt erfolgte. Beurkundete Todesfälle können maximal bis zum Datum des Vortages (aktuelles Datum - 
1 Tag) in die Exportdatei übernommen werden. Die beiden bundesweiten Anbieter der Software zur Datenverarbeitung in Standesämtern sowie das HSL haben die Programme entsprechend angepasst und damit eine wöchentliche Datenübermittlung ermöglicht.

\section{Datenqualität und Auswertungsprozesse}

Die Daten ermöglichen aufgrund der Einzelfallerfassung verschiedenste Stratifizierungen, zum Beispiel hinsichtlich Altersgruppen, Regionen und Zeit. Damit wird es möglich, Zusammenhänge zwischen erhöhten Todesfallzahlen und bekannten Gesundheitsgefahren, wie zum Beispiel Hitzewellen, zu explorieren und zu bewerten.

Die Auswertung der Daten im HLPUG erfolgt mit dem Statistikprogramm STATA. Wöchentlich werden die Todesfälle in Hessen nach sieben Altersgruppen sowie je Kalenderwoche nach Versterbekreis zusammengestellt. Die Vollzähligkeit wird anhand der Kontrolldatei und des durchschnittlichen Beurkundungsverzugs (siehe unten) abgeschätzt.

\section{Zeitnähe der im HLPUG zur Verfügung stehenden Daten}

Die im HLPUG eingehenden Datensätze sind zunächst nicht vollzählig, werden jedoch mit den Folgemeldungen ergänzt. Dieser Verzug erklärt sich durch einen:

Übermittlungsverzug (ÜbermittlungsCompliance der Standesämter): Übermittlungsverzögerungen beziehen sich auf ganze Datenpakete. Ursachen dafür können zum Beispiel EDV-Probleme, Krankheit oder ein Vergessen der Übermittlung der oder des Verantwortlichen sein.

\section{und/oder}

Beurkundungsverzug: Beurkundungsverzögerungen für einzelne Todesfälle entstehen, wenn zum Beispiel die für die Beurkundung benötigten Unterlagen nicht vollständig sind.

In der Folgewoche wird die Vollzähligkeit der Daten auf etwa 70 bis $80 \%$ ergänzt, mit der Übermittlung in der zweiten Folgewoche auf etwa 90\%.

Zur epidemischen Phase der A/H1N1Pandemie 2009/10 hatte sich die wöchent-

Bundesgesundheitsbl $2011 \cdot 54: 867-874 \quad$ DOI 10.1007/s00103-011-1298-x

(C) Springer-Verlag 2011

\section{H. Uphoff · S. Geis · A. Wirtz · A.M. Hauri}

Zeitnahe Erfassung und Übermittlung von Todesfällen in Hessen. Eine erste Einschätzung zur Influenza-A/H1N1v-Pandemie

\section{Zusammenfassung}

Die Erfassung von Todesfällen ist ein wichtiges epidemiologisches Instrument, um den Verlauf, das Ausmaß, betroffene Altersgruppen und die Schwere von Gesundheitsgefahren einschätzen zu können. Dennoch sind in Deutschland weder auf Landes- noch auf Bundesebene geeignete Daten oder Auswertungen zeitnah zugänglich. Im vorliegenden Beitrag werden Aufbau und Struktur eines Surveillance-Systems zur zeitnahen Erfassung von Todesfällen in Hessen (ZETH) und erste Erfahrungen während der Influenza-A/ H1N1v-Pandemie 2009/2010 dargestellt. Während des Aufbaus des ZETH wurde 2008 auf eine wöchentliche Übermittlung der bei den Standesämtern elektronisch erfassten Todesfälle (das heißt etwa $95 \%$ aller Todesfälle) an das Statistische Landesamt umgestellt. Das Landesamt stellt nun - ebenfalls wöchentlich - diese Daten dem Hessischen Landesprüfungs- und Untersuchungsamt im Ge- sundheitswesen (HLPUG) in todesfallbasierter datenschutzkonformer Form zur Verfügung. Während der Influenza-Pandemie gestattete das ZETH die zeitnahen Einschätzungen der Übersterblichkeit nach Altersgruppen mit einer zweiwöchigen Verzögerung. Es zeigte sich eine geringe Zunahme der Todesfälle über das Erwartungsniveau in den Altersgruppen von 15 bis 34,35 bis 49 und 50 bis 59 Jahren. Der Zusammenhang zum zeitlichen Verlauf und zur Intensität der Erkrankungswelle war jedoch nicht stark ausgeprägt, sodass eine Übersterblichkeit, die offensichtlich über die im Meldewesen erfassten Influenza-Todesfälle (21 für Hessen) hinausgeht, nicht deutlich wurde.

\section{Schlüsselwörter}

Influenza · Pandemie · A/H1N1 .

Todesfälle $\cdot$ Surveillance

\section{Timely registration of fatalities in the state of Hessen. Experiences during the influenza A/H1N1 pandemic}

\section{Abstract}

Timely registration of fatalities is important for the assessment of course, extent, risk of age groups, and magnitude or severity of health threats. Nevertheless, timely data of casualties are not available on the state or national level. The current paper describes the implementation and structure of a surveillance system for the timely registration of casualties in the state of Hesse (Germany) and the experience obtained during the pandemic 2009/10. The delay of the casebased registration appears tolerable and after 2 weeks more than $80 \%$ of all deaths for a given week are registered. In 2008 , the forwarding of the electronically registered data from the registry offices ( $95 \%$ of all cases) to the state statistical office (HSL) had been accelerated from a monthly to a weekly base. The HSL provides - on a weekly basis - this case-based data in accordance with data protection rules to the Hesse State Health Office (HLPUG, "Hessischer Landesprüfungs- und Untersuchungsamt im Gesundheitswesen"). During the pandemic, the data allowed assessment of the excess mortality with a delay of 2 weeks. No significant excess mortality was apparent; however, a slight increase was observed in the age groups 15-34, 3549 , and 50-59. Correlation of time with the severity of the $A / H 1 N 1 v$ epidemic was not very strong. Hence, the data did not indicate an excess significantly exceeding the number of death cases registered with the mandatory reporting system of 21 cases for Hesse.

\section{Keywords}

Influenza · Pandemics · A/H1N1 .

Mortality · Surveillance 


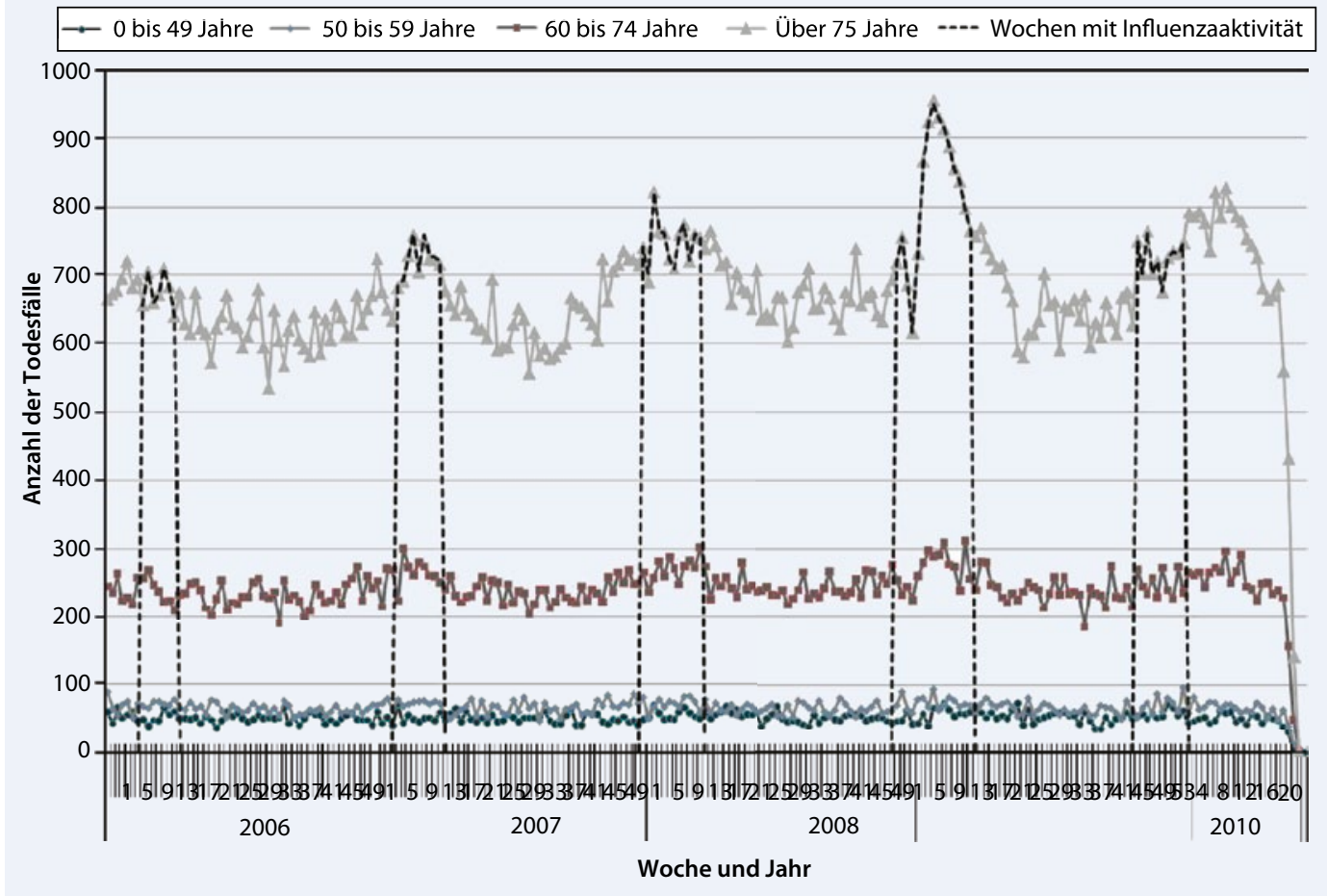

Abb. $1<$ Im hessischen Surveillance-System ZETH registrierte Todesfälle (nach Woche und Altersgruppe) und Zeiträume mit erhöhter Influenza-Aktivität laut der Arbeitsgemeinschaft Influenza (AGI) (siehe Text); 1. KW 2006 bis 16. KW 2010

liche Datenübermittlung an das HSL und von dort weiter an das HLPUG stabilisiert. Es konnten damit wöchentliche Routineauswertungen vorgenommen werden. Im Rahmen des EUROMOMOProjektes (www.euromomo.eu/) wurden auch wöchentlich aggregierte Daten an das Statens Serum Institut in Dänemark als Hauptprojektpartner übermittelt. Im Folgenden werden verschiedene während der Pandemie durchgeführte Routineund Sonderauswertungen dargestellt und bewertet $[16,17]$.

\section{Erfahrungen und Aus- wertungsergebnisse zur A/ H1N1-Pandemie 2009/2010}

\section{Jahreszyklus bei wochenweiser Betrachtung nach Altersgruppen}

In - Abb. 1 sind die von der 1. Kalenderwoche (KW) 2006 bis zur 16. KW 2010 (Stichtag 4.5.10) erfassten Todesfälle in Hessen in vier Altersgruppen dargestellt. Es wird der insbesondere in den höheren Altersgruppen (6o bis 74 Jahre und $\geq 75$ Jahre) zunehmend ausgeprägte zyklische Jahresgang mit den Wintergipfeln deutlich. Die durch senkrechte gestrichelte Abgrenzungen markierten Phasen weisen Zeiträume mit erhöhter Influenza-Aktivität aus. Diese wurden anhand des Jahresabschlussberichtes der Arbeitsgemeinschaft Influenza (AGI) am Robert Koch-Institut (RKI) als Zeiträume mit relevanter Viruszirkulation und Morbiditätserhöhung von über mehr als zwei Wochen definiert.

Vermehrte Todesfälle während der Phasen einer erhöhten Influenza-Aktivität sind insbesondere in den höheren Altersgruppen zu Beginn 2009 offensichtlich. In dieser Phase wurde eine ausgeprägte Aktivität der Influenza A/ $\mathrm{H}_{3} \mathrm{~N}_{2}$ beobachtet. Auch im ersten Quartal des Jahres 2010 sind insbesondere bei den über 75-Jährigen hohe Sterbezahlen erkennbar.

Die pandemische Influenza A/H1N1v erreichte eine intensive, mit erhöhter Morbidität einhergehende Zirkulation auf Bevölkerungsebene etwa zwischen der 43. und 53. KW 2009. Betroffen waren laut RKI insbesondere jüngere Altersgruppen [18].

\section{Vergleichende Betrachtungen zum Verlauf der wöchentlichen Sterbezahlen}

An - Abb. 2 soll untersucht werden, ob in den verschiedenen Altersgruppen beobachtete Veränderungen der Sterbehäufigkeit in einem zeitlichen Zusammenhang mit der Zirkulation der pandemischen Influenzaviren aufgetreten sind.
Hierzu wurde der prozentuale Anteil der wöchentlichen Todesfälle nach Altersgruppen an einem fixen Wochendurchschnitt dargestellt. Der Wochendurchschnitt wurde anhand der Todesfälle im Zeitraum zwischen der 1. KW 2006 und der 53. KW 2009 berechnet. Der prozentuale Anteil wurde als gleitender Durchschnitt über fünf Wochen berechnet. Zum Vergleich sind in $\bullet$ Abb. 2 die nach Infektionsschutzgesetz (IfSG) bundesweit gemeldeten Influenza-Erkrankungsfälle als \%。 der Summe aller gemeldeten Fälle sowie die bundesweit gemeldeten Influenza-Todesfälle enthalten. Der anhand dieser Daten beschriebene Verlauf der A/ H1N1-Pandemie stimmt sehr gut mit den Daten der Arbeitsgemeinschaft Influenza (AGI) (http://influenza.rki.de/Wochenberichte.aspx) und den hessischen Meldefällen nach IfSG überein.

Die Zahl der nach IfSG registrierten Todesfälle nimmt in der 44 . KW mit dem Anstieg der nach IfSG gemeldeten Erkrankungsfälle zu, erreicht ihr Maximum etwa gemeinsam mit dem Maximum der Meldefälle, nimmt aber deutlich verzögert $\mathrm{ab}$ und erreicht erst Ende Februar 2010 wieder ein sporadisches Niveau. Im Folgenden werden die in $\bullet$ Abb. 2 dargestellten Kurven für die einzelnen Altersgruppen betrachtet. 


\begin{abstract}
Abb. 2 $>$ In Hessen nach Infektionsschutzgesetz (IfSG) gemeldete Influenza-Erkrankungsfälle als wöchentlicher Anteil (Tausendstel) der Summe aller zwischen der KW. 222009 bis zur KW. 62010 gemeldeten Influenza-Fälle (linke Achse); wöchentlicher Anteil (als gleitendes Mittel über fünf Wochen in Hundertsteln) der im hessischen Surveillance-System ZETH erfassten Todesfälle an einem fixen Wochendurchschnitt (nach Altersgruppen, für Hessen, 1. KW 2009 bis 16. KW 2010). Der fixe Wochendurchschnitt wurde anhand der Todesfälle im Zeitraum zwischen der 1. KW 2006 bis zur 53. KW 2009 berechnet (linke Achse); bundesweit gemeldete Todesfälle (rech-
\end{abstract} te Achse)
Klein- und Schulkinder. Bei den Klein(null bis vier Jahre) und Schulkindern (fünf bis 14 Jahre) sind aufgrund der geringeren Zahlen die Abweichungen vom Mittelwert auch nach der Glättung beträchtlich. So liegt der Durchschnitt der Todesfälle je Woche für die Null- bis Vierjährigen bei 4,1; für die Altersgruppe der fünf- bis 14-Jährigen bedeutet ein zusätzlicher Fall pro Woche einen Anstieg um $100 \%$. Weder positive noch negative $\mathrm{Ab}$ weichungen können eindeutig bestimmten Ereignissen zugeordnet werden.

Altersgruppe 15 bis 34 Jahre. In der Altersgruppe der 15 - bis 34 -Jährigen mit einer mittleren wöchentlichen Anzahl von 8,5 im ZETH registrierten Todesfällen zeigen sich ebenfalls noch erhebliche Abweichungen vom Durchschnitt. Ein Anstieg von leicht unterdurchschnittlichen auf etwas überdurchschnittliche Werte ist bereits deutlich vor der Epidemie-Phase erkennbar. Er setzt sich aber während der Epidemie noch fort. Die höchste Fallzahl wird in der 46. KW mit 17 Todesfällen registriert.
Altergruppe 35 bis 49 Jahre. In der $\mathrm{Al}-$ tersgruppe der 35- bis 49-Jährigen liegt der Wochendurchschnitt bei den Todesfällen bei 36,6. Hier ist eine moderate Erhöhung der Fallzahl in einem möglichen zeitlichen Zusammenhang mit den nach IfSG erfassten Todesfällen erkennbar. Die höchste Zahl mit 52 Todesfällen wird in der 49. KW registriert.

Höhere Altersgruppen. In den höheren Altersgruppen (50 bis 59, 60 bis 75 und $>75$ Jahre) sind die saisonal bedingten Abweichungen vom Wochendurchschnitt erkennbar. Darüber hinaus fallen erhöhte Werte zu Beginn des Jahres 2009 auf. Sie stehen in einem engen zeitlichen Zusammenhang mit der ausgeprägten Influenza-A/ $\mathrm{H}_{3} \mathrm{~N}_{2}$-Welle. Während der epidemischen Phase der A/H1N1-Pandemie ist lediglich für die Altersgruppe der 50bis 59-Jährigen eine geringe, nicht ungewöhnliche Zunahme der Sterbefälle im Zeitraum erhöhter Todesfallmeldungen nach IfSG - mit einem Spitzenwert von 88 Todesfällen in der $47 . \mathrm{KW}$ - zu erkennen.

Zusammenfassend ist festzustellen, dass in den drei Altersgruppen 15 bis
34 Jahre, 35 bis 49 Jahre und 50 bis 59 Jahre Erhöhungen der Todesfallzahlen in einem plausiblen zeitlichen Zusammenhang zur Pandemie zu erkennen sind. In allen drei Altersgruppen werden während der $\mathrm{A} /$ $\mathrm{H}_{1} \mathrm{~N}_{1}$-Pandemie erhöhte Werte beobachtet. Gleiche oder höhere Werte werden im Zeitraum 2006 bis Oktober 2010 in weniger als 1,9\% aller Wochenwerte in der Altersgruppe 15 bis 34 Jahre erreicht, in 1,4\% der Altersgruppe 35 bis 49 Jahre und in $1,4 \%$ der Altersgruppe 50 bis 59 Jahre. Die im ZETH gemachten Beobachtungen korrelieren mit der Tatsache, dass etwa $68 \%$ der bundesweit im Zusammenhang mit einer $\mathrm{A} / \mathrm{H}_{1} \mathrm{~N}_{1 v}$-Infektion nach IfSG gemeldeten Todesfälle diese Altersgruppen betrafen.

\section{Todesursachenermittlung für zwei Peaks im Jahr 2009 (je zwei Wochen) bei Frauen der Altersgruppe 20 bis 40 Jahre}

Aufgrund des Risikos, die eine saisonale $[19,20]$ und insbesondere eine pandemische Influenza für Schwangere birgt, wurden die Todesfälle bei 20- bis 40-jährigen Frauen gesondert betrachtet (• Abb. 3). 


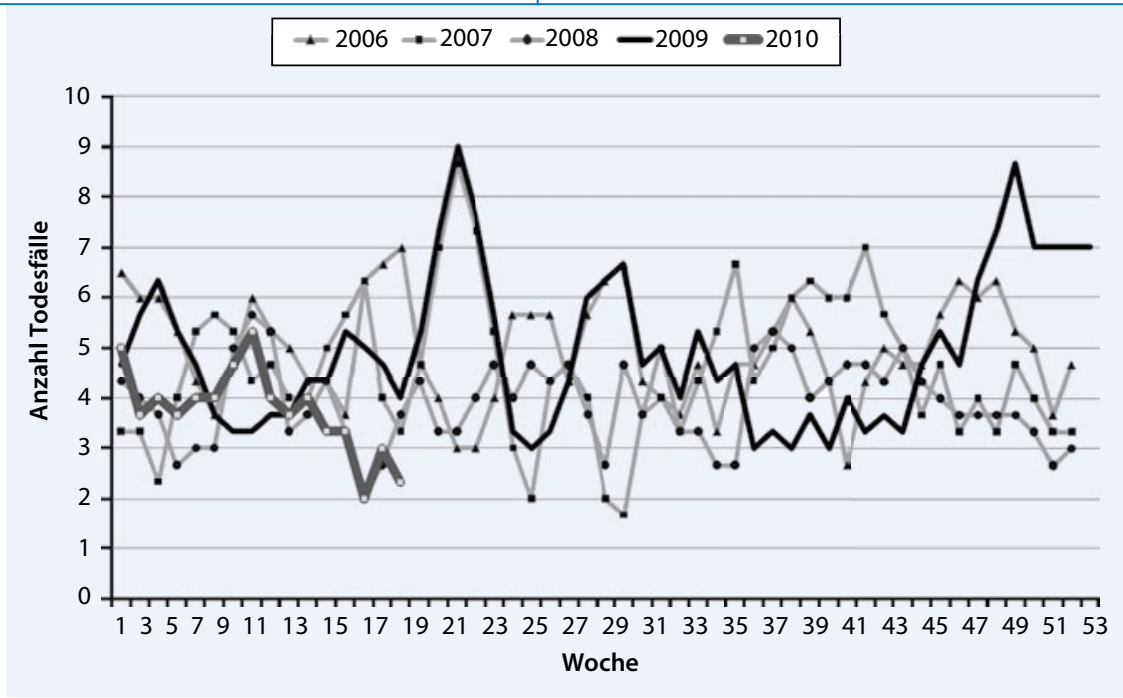

Abb. $3 \Delta$ Im hessischen Surveillance-System ZETH registrierte Todesfälle bei Frauen in der Altersgruppe 20 bis 40 Jahre (gleitendes Fünf-Wochen-Mittel), nach Wochen, 2006 bis 16. KW 2010

Es zeigt sich in dieser Gruppe ein Anstieg der Todesfallzahlen in einem zeitlichen Zusammenhang mit der pandemischen Influenza mit einem Spitzenwert von zehn Fällen in der 48. KW 2009 (Wochendurchschnitt 4,6 Fälle). Vergleichbare Spitzenwerte wurden im Beobachtungszeitraum zwischen der 1. KW 2006 bis zur 16. KW 2010 in 2,4\% der Wochen erreicht oder überschritten.

Ein zeitlich naheliegender vergleichbarer Peak wurde in der 18. und 19. KW 2009 erreicht. Die Todesursachen bei den in diesen zwei Wochen verstorbenen Frauen wurden mit denen der in der 48 . und 49. KW 2009 verstorbenen verglichen (die Todesursachen bei den jeweils 20 verstorbenen Frauen wurden von den Gesundheitsämtern zur Verfügung gestellt). In der 18. und 19. KW 2009 wurden in 19 Fällen bösartige Neubildungen, schwerste Grunderkrankungen, Unfall oder Suizid als Todesursache angegeben. Bei einem Todesfall wurde auf der Sterbeurkunde eine Pneumonie, vermutlich im Zusammenhang mit einer Kardiomyopathie, genannt.

In der 48. und 49. KW 2009 wurden in 14 Todesfällen bösartige Neubildungen, schwerste Grunderkrankungen, Unfall oder Suizid als Todesursachen registriert. Bei drei Todesfällen war keine Ursache ermittelbar, oder es wurde ein „natürlicher Tod" angegeben. Für einen Todesfall war eine Pneumonie unklarer Genese bei einem metastasierenden Mammakarzinom attestiert, und in zwei Fällen wur- de eine Influenza-A-Pneumonie genannt. In einem dieser Fälle stand die InfluenzaA-Pneumonie in einem eindeutigen ursächlichen Zusammenhang mit dem Tod. Dieser Fall war auch als Influenzatodesfall nach IfSG gemeldet worden. Der zweite Fall wurde nicht gemeldet, und aufgrund der ebenfalls auf dem Totenschein angegebenen Transplantat-gegen-Wirt-Reaktion (Lunge) bleibt die Rolle der Influenzainfektion unklar.

\section{Diskussion}

\section{System}

Lebensbedrohliche Gesundheitsgefahren sind weltweit stärker ins Bewusstsein gerückt $[8,9,10,11]$. Die Analyse von Todesfällen als eindeutige Ereignisse bei routinemäßig vollständiger Erfassung ist ein wichtiges epidemiologisches Instrument $[17,21]$. Das für Hessen umgesetzte Projekt ist ein Kompromiss zwischen den derzeit bestehenden datentechnischen, datenschutzrechtlichen, gesetzlichen und organisatorischen Gegebenheiten und den Ansprüchen an die zeitliche Nähe und räumlich-zeitliche Auflösung. Es wurde den wesentlichen Anforderungen an die Auflösung und Zeitnähe Rechnung getragen und auf die direkte Einbeziehung der mutmaßlichen Todesursachen verzichtet.

Das hier beschriebene System erlaubt eine Bewertung der Originaldaten mit einer Verzögerung von etwa zwei Wochen, da erst dann eine ausreichende Voll- zähligkeit erreicht wird. Dennoch können erste Signale für eine erhöhte Sterblichkeit zu einem Zeitpunkt aufgefangen werden, an dem die vertraulichen Teile der Sterbeurkunden im Gesundheitsamt ankommen oder vorliegen. Das Gesundheitsamt hat dann die Möglichkeit, auch die dort vorliegenden Todesursachen in die Überlegungen zu den Ursachen einer Exzessmortalität einzubeziehen.

Auf Berechnungen statistischer Signifikanzen, die abhängig von den willkürlich gewählten Altersgruppen, den betrachteten Zeiträumen, dem gewählten Modell für Erwartungswerte, der Methode etc. variieren, wurde verzichtet. Auch im Euro-MOMO-Projekt werden zunächst nur die Standardabweichungen vom modellierten Erwartungswert betrachtet $[16,17]$.

\section{Pandemie}

Die zeitnahe Erfassung der Todesfälle in Hessen hat sich während der Pandemie als wichtige Ergänzung zu den über das IfSGMeldewesen erfassten Daten erwiesen. Da die Vollzähligkeit mithilfe der „Kontrolldatei“ abgeschätzt werden konnte, waren erste Bewertungen mit etwa zweiwöchiger Verzögerung möglich. Dabei zeigte sich bereits während der Pandemie, dass keine bedeutsame, um ein Mehrfaches über die nach IfSG erfassten Todesfälle hinausgehende Übersterblichkeit aufgetreten ist.

Problematischer als das Erkennen einer ungewöhnlichen Abweichung in der Sterbehäufigkeit ist deren Zuordnung zu einer möglichen Ursache. In erster Linie kann die Zuordnung bei einer Betrachtung der Gesamttodesfälle ohne nähere Angaben zur Todesursache über einen zeitlichen Zusammenhang im Sinne einer plausiblen „Dosis-Wirkungs-Beziehung" und - bei häufigerem Auftreten des mutmaßlich ursächlichen Ereignisses - zusätzlich über eine Korrelation zwischen den Ereignissen und der veränderten Sterbehäufigkeit gefestigt werden. Insgesamt sind die Zusammenhänge komplex, und es existiert kein umfassendes Modell aller möglichen und zeitlich konkurrierenden Einflüsse.

Für die offensichtliche Übersterblichkeit bei den höheren Altersgruppen, insbesondere den über 75-Jährigen, zu Beginn des Jahres 2009 ist ein plausibler $\mathrm{Zu}$ - 
sammenhang zur Influenza-A/H3 N2-Aktivität offensichtlich. Dieser gründet sich auf dem engen zeitlichen Zusammenhang zur Erkrankungswelle und zur intensiven Viruszirkulation, dem typischen Verlauf, den betroffenen Altersgruppen sowie den diesbezüglichen Exzessen an Todesfällen über viele Jahre [10, 22]. Die Korrelationen sind eng und im Sinne einer „DosisWirkungs-Beziehung " plausibel, insbesondere wenn die Influenza nicht undifferenziert, sondern getrennt nach Typen und Subtypen betrachtet $[23,24]$ und die Intensität der Influenzazirkulation insbesondere in den höheren Altersgruppen einbezogen wird. Die ebenfalls recht hohen Sterbezahlen bei den über 75-Jährigen im ersten Quartal 2010 stehen nicht im Zusammenhang mit einer Influenzazirkulation. Es ist nicht geklärt, ob niedrige Temperaturen [25] oder auch andere, insbesondere virale Infektionen, die in der kühlen lichtarmen Jahreszeit besonders häufig sind (zum Beispiel RS-, Metapneumo-, Parainflunza- oder auch Norooder Rotaviren), Einfluss auf die erhöhte Sterbehäufigkeit hatten. Tatsächlich wurden in diesem Zeitraum vermehrt RS-Viren und Metapneumoviren nachgewiesen, aber auch relativ niedrige Temperaturen registriert [26].

Eine im zeitlichen Verlauf plausible Übereinstimmung zwischen der intensiven Zirkulation des pandemischen A/ $\mathrm{H}_{1} \mathrm{~N}_{1}$-Virus und einer geringfügigen Erhöhung der Sterbefallzahl ist nur in den mittleren Altersgruppen von 15 bis 59 Jahre zu erkennen. In dieser Altersgruppe wurde auch die Mehrzahl der Todesfälle (68\%) über das bundesweite Meldewesen registriert. Für Kinder war, abweichend von einer vorläufigen Auswertung länderübergreifender gepoolter Daten [17], anhand der hessischen Daten keine Übersterblichkeit erkennbar.

Ein plausibler Zusammenhang zwischen den im ZETH erfassten Todesfällen und dem $\mathrm{A} / \mathrm{H}_{1} \mathrm{~N}_{1}$-Epidemieverlauf beziehungsweise den nach IfSG gemeldeten Todesfällen lässt sich aufgrund der geringen Todesfallzahlen und des oft deutlichen zeitlichen Abstandes zwischen Erkrankungs- und Todesdatum nicht festigen. Auch die Stichprobenuntersuchung zu den Todesursachen bei Frauen zwischen 20 und 40 Jahren ergab keine zwin- genden Hinweise auf eine Übersterblichkeit, die deutlich über die bereits nach IfSG gemeldeten Todesfälle hinausgeht.

Bei der geringen Zunahme der Todesfälle in der Altersgruppe 15 bis 59 Jahre und der durchschnittlichen Fallzahl in dieser Altersgruppe je Woche für Hessen von 112,4 , erscheint die hier über das IfSG erfasste Zahl von 21 Todesfällen plausibel.

Bei einer selektiven Erfassung von Todesfällen (zum Beispiel wie im Meldewesen durch eine Verknüpfung mit einem Labornachweis von $\mathrm{H}_{1} \mathrm{~N} 1 \mathrm{v}$ ) ist zunächst völlig unklar, ob es sich um ohnehin erwartete oder um „Exzess“-Todesfälle handelt. Eine Verbindung mit einem Exzess ist wahrscheinlicher, wenn das Ereignis (hier Labornachweis von $\mathrm{H}_{1} \mathrm{~N} 1 \mathrm{v}$ ) in einer ungewöhnlichen Häufung den Tod bei Personen herbeiführt, die sonst nicht gestorben wären. Da in dem hier betrachteten Alterssegment Todesfälle aufgrund akuter Infektionen selten sind (bösartige Neubildungen, Unfall, Suizid und schwere chronische Grunderkrankungen sind in dieser Altersgruppe dominant), ist die Wahrscheinlichkeit, dass es sich bei den Meldefällen mit entsprechender Symptomatik um zusätzliche $\mathrm{A} / \mathrm{H}_{1} \mathrm{~N}_{1}$-Sterbefälle handelt, hoch.

\section{Fazit und Ausblick}

Gegenüber bisheriger Systeme zu Erfassung monatlicher Sterbezahlen (mit einer Verzögerung von etwa sechs Monaten und mehr) stellt das hessische Surveillance-System eine wesentliche Verbesserung und Beschleunigung dar, auch wenn ein direkter Bevölkerungsbezug der mit ihm erhobenen vorläufigen Todesfallzahlen problematisch ist. Insgesamt birgt die zeitnahe Erhebung der Todesfälle ein großes Potenzial und ist eine wichtige Ergänzung der Datenbasis zur Einschätzung von Gesundheitsgefahren. Auch ohne lange historische Zeitreihen und Modelle für entsprechende Erwartungswerte hat sich die zeitnahe Erfassung der Todesfälle als sehr hilfreich erwiesen und die Einschätzung der Gesundheitsgefahren während der Pandemie verbessert.

Eine drastische Zahl an Exzesstodesfällen, die die im Meldewesen registrierten Todesfälle um ein Vielfaches überschrei- tet, ist im unmittelbaren zeitlichen Zusammenhang mit dem Zirkulieren von A/H1N1v nicht erkennbar. Eine über die mit dem Meldewesen erfassten Todesfälle hinausgehende Zahl von Todesfällen im Zusammenhang mit A/H1N1v ist aber dennoch nicht auszuschließen.

Verbesserungsbedarf wird insbesondere bei der zeitnahen Datenübermittlung gesehen, zum Beispiel über eine weitere Automatisierung des Prozesses. Darüber hinaus sollen die Möglichkeiten zur Vollzähligkeitsschätzung verbessert und weiter ausgebaut werden.

Die Einbeziehung weiterer historischer Daten wird derzeit geprüft, um belastbare Modelle zur Abschätzung von „Normal-“ beziehungsweise Erwartungswerten zu erstellen und mögliche Einflussfaktoren auf die Sterblichkeit besser bewerten zu können.

Das Nutzungspotenzial soll durch die Erschließung und regelmäßige Bewertung weiterer Vergleichsdaten, zum Beispiel zu Hitzewellen und Feinstaubbelastung, weiter ausgeschöpft werden. In diesem Zusammenhang soll auch die räumliche Auflösung für Routineauswertungen verfeinert werden.

\section{Korrespondenzadresse}

\section{Dr. H. Uphoff}

Hessisches Landesprüfungsund Untersuchungsamt im

Gesundheitswesen

Wolframstr. 33, 35683 Dillenburg

Helmut.Uphoff@hlpug.hessen.de

Danksagung. Wir möchten folgenden Personen für die Unterstützung des Projektes danken: Herrn Meireis (HMdl), Herrn Emanuel (i.R.), Frau Schmidt-Wahl (HSL), Frau Müller (Mitarbeiterin des Datenschutzbeauftragten Hessen), Frau Schumacher (Standesamt Wiesbaden), Herrn Broos (Standesamt Frankfurt), Herrn Heinemann und Herrn Thiemer als Vertreter der Software-Hersteller sowie allen Mitarbeitern und Mitarbeiterinnen der Standesämter und des Statistischen Landesamtes, die durch ihr Engagement zum Gelingen des Projektes beitragen.

Interessenkonflikt. Der korrespondierende Autor gibt an, dass kein Interessenkonflikt besteht.

\section{Literatur}

1. Arbeitsgemeinschaft Influenza. Abschlussbericht der Influenzasaison 1994/95 bis 2007/08. http://influenza.rki.de/Saisonbericht.aspx

2. Statistisches Bundesamt (1991) Allgemeine Sterbetafel 1986/88. Wirtsch Stat 6:371-373 
3. Zucs P, Buchholz U, Haas W, Uphoff H (2005) Influenza associated excess mortality in Germany, 1985-2001. Emerg Themes Epidemiol 2:6

4. Robert Koch-Institut (2007) Zur Influenza-assoziierten Mortalität in Deutschland. Epidemiol Bull 35:325-327

5. Statistisches Bundesamt (2007) Arbeitsunterlagen Statistisches Bundesamt, N30. Wiesbaden

6. Heudorf U, Stark S (2004) Gesundheitsgefahren durch extreme Hitze - Prävention ist notwendig - Konsequenzen aus der Hitzeperiode im August 2003. Hess Arztebl 7:420

7. Robert Koch-Institut (2004) Hitzewellen und extreme Klimaereignisse - Eine Herausforderung für das Gesundheitswesen. Epidemiol Bull 25:200201

8. Koppe C, Jendritzky G (2004) Die Auswirkungen der Hitzewellen 2003 auf die Mortalität in BadenWürttemberg (Zwischenbericht I des Projekts „Klimawandel - Auswirkungen, Risiken, Anpassung KLARA"). In: Gesundheitliche Auswirkungen der Hitzewelle im August 2003. Sozialministerium Baden-Württemberg, Stuttgart

9. Economopoulou A, Dominguez M, Helynck B et al (2009) Atypical Chikungunya virus infections: clinical manifestations, mortality and risk factors for severe disease during the 2005-2006 outbreak on Reunion. Epidemiol Infect 137:534-541

10. Ramchurn SK, Goorah SS, Makhan M, Moheeput K (2008) Excess mortality as an epidemic intelligence tool in chikungunya mapping. Euro Surveill 13:1-13

11. Crofts J (2003) Current influenza activity in the United Kingdom: A/Fujian/411/2002 (H3N2)-like strains circulating in the community, week 44. Euro Surveill 7

12. Kovats S, Wolf T, Menne B (2004) Heatwave of August 2003 in Europe: provisional estimates of the impact on mortality. Euro Surveill 8(11)

13. Fouillet A, Rey G, Wagner V et al (2008) Has the impact of heat waves on mortality changed in France since the European heat wave of summer 2003? A study of the 2006 heat wave. Int J Epidemiol 37:309-317

14. Miller MA, Viboud C, Olson DR et al (2008) Prioritization of influenza pandemic vaccination to minimize years of life lost. J Infect Dis 198:305-311

15. Expertengruppe,Influenza-Pandemieplanung" am Robert Koch-Institut. Nationaler Pandemieplan Teil III. http://www.rki.de/cln_100/nn_200120/DE/ Content/InfAZ/I/Influenza/influenzapandemieplan_III,templateld=raw, property=publicationFile.pdf/influenzapandemieplan_III.pdf

16. Mazick A, on behalf of the participants of a workshop on mortality monitoring in Europe (2007) Monitoring excess mortality for public health action: potential for a future European network. Euro Surveill 12:3107

17. Mazick A, Gergonne B, Wuillaume F et al (2010) Higher all-cause mortality in children during autumn 2009 compared with the three previous years: pooled results from eight European countries. Euro Surveill 15(5)

18. Krause G, Buchholz U (2010) Rückblick: Epidemiologie und Infektionsschutz im zeitlichen Verlauf der Influenzapandemie (H1N1) 2009. Epidemiol Bull 21:191-197

19. Jamieson DJ, Honein MA, Rasmussen SA et al (2009) H1N1 2009 influenza virus infection during pregnancy in the USA. Lancet 374:451-458

20. Rasmussen SA, Jamieson DJ, Bresee JS (2008) Pandemic influenza and pregnant women. Emerg Infect Dis 14:95-100
21. Hutwagner L, Thompson W, Seeman GM, Treadwell T (2003) The bioterrorism preparedness and response Early Aberration Reporting System (EARS). J Urban Health 80:i89-i96

22. Uphoff H, Stilianakis NI (2004) Influenza-associated excess mortality from monthly total mortality data for Germany from 1947 to 2000. Methods Inf Med 43:486-492

23. Thompson WW, Shay DK, Weintraub E et al (2003) Mortality associated with influenza and respiratory syncytial virus in the United States. JAMA 289:179186

24. Simonsen L, Clarke MJ, Williamson GD et al (1997) The impact of influenza epidemics on mortality: introducing a severity index. Am J Public Health 87:1944-1950

25. Anderson BG, Bell ML (2009) Weather-related mortality: How heat, cold, and heat waves affect mortality in the United States. Epidemiology 20:205213

26. Niedersächsisches Landesgesundheitsamt. Surveillance für Influenza und andere akute respiratorische Erkrankungen in Niedersachsen. Jahresbericht Saison 2009/2010. http://www.nlga. niedersachsen.de/live/live.php?navigation_ id=27105\&article_id=19408\&_psmand=20 